\title{
A Monte Carlo simulation study for
}

\section{Kolmogorov-Smirnov two-sample test under the precondition of heterogeneity : upon the changes on the probabilities of statistical power and type I error rates with respect to skewness measure}

\author{
Ötüken Senger ${ }^{1}$ and Ali Kemal Çelik ${ }^{2}$
}

\begin{abstract}
Skewness and kurtosis are adopted by many statisticians as the contraventions of parametric statistics. Therefore, using nonparametric tests would give more proper results for skewed and kurtic series. Many observations also suggest that skewness provokes the loss of power for statistical tests. This paper aims to investigate the impact of skewness on statistical power. For this purpose, the paper takes hold of nine different distributions on Fleishman's power function when skewness measures are $1,75,1,50,1,25,1,00,0,75,0,50,0,25,0,00,-0,25$ and kurtosis measure is 3,75 , simultaneously. The investigation concentrates on Kolmogorov-Smirnov two-sample test and considers the significance level $(\alpha)$ as 0,05 . This paper runs totally 32 representative sample size simulation alternatives, involving four small and equal; twelve small and different; four large and equal; and twelve large and different sample sizes. The Monte Carlo simulation study takes standard deviation ratios as 2, 3 and 4 under the precondition of heterogeneity. According to the results of equal sample sizes, no significant change are observed on the possibility of Type I error for Kolmogorov-Smirnov
\end{abstract}

1 Kafkas University, Department of Numerical Methods, Kars, Turkey.

2 Atatürk University, Department of Numerical Methods, Erzurum, Turkey.

Article Info: Received : July 15, 2013. Revised : August 15, 2013

Published online : December 1, 2013 
tests, when the skewness measures decrease from 1,75 to $-0,25$. For both small and large small sizes, the power of the corresponding test decreases when the coefficient of skewness decreases.

\section{Mathematics Subject Classification: 62G08, 62GXX}

Keywords: Power of test, Skewness, Kolmogorov-Smirnov two-sample test, Monte Carlo simulation study

\section{Introduction}

Parametric tests have pre-conditions of normal distribution and homogeneity of variance. Normal distribution is a kind of symmetric distributions being optimized mathematically and it offers a fine mathematical curve of frequency distributions for behavioral sciences [1]. Homogeneity variance is an assumption that populations from which samples have been drawn have equal or similar variances [2]. Skewness is one of the two occasions that any sample distribution principally diverges from normality and for many researchers it causes the loss of statistical power [3]. Nonparametric tests are very often more advantageous than parametric tests when one or both of these pre-conditions are not satisfied.

Skewed and kurtic data sets are practically experienced more than normal distributed data. Surely, researchers seek to know whether statistical tests being used have sufficient power. Statistical power of a test varies depending on sample size, skewness, kurtosis, standard deviation and mean ratios. Thereof, researchers are expected to make decisions on which parametric and nonparametric tests they will use with respect to these concerning indicators.

This paper investigates how skewness effects statistical power of nonparametric tests and handles Kolmogorov-Smirnov two-sample test (KS-2). This paper concentrates on heterogeneity of variance pre-condition and utilizes from skewed and kurtic data simultaneously, hence it may differ from recent studies that evaluate the statistical power of nonparamatric tests.

\section{Kolmogorov-Smirnov Two-Sample Test}

There are several tests available to determine if a sample comes from a normally distributed populations, that include the Kolmogorov-Smirnov test, Anderson-Darling test, Cramer-von Mises Test, Shapiro-Wilk test and Shapiro-Francia test [4]. The Kolmogorov-Smirnov test is a method for comparing the distributions of two independent groups that has virtually disappeared from applied research and introductory statistics books for the social sciences [5]. The Kolmogorov-Smirnov test is important in nonparametric stastistical inference, 
while the limiting distribution of the Kolmogorov-Smirnov statistic under the null hypothesis is well known and has been derived by several different methods. The limiting distribution of this statistic under the alternative hypothesis is unknown so that it is not possible, in general, to compute the power of the Kolmogoro-Smirnov test [6].

The classical one-dimensional Kolmogorov-Smirnov test is a non-parametric statistic for comparing two empirical distributions which defines the largest absolute difference between the two cumulative distribution functions as a measure of disagreement and is used to test the null hypothesis $F=G$ against the alternative $F>G$ where $F$ and $G$ are distribution functions. If the random variables $X$ and $Y$ correspond to $F$ and $G$, respectively, then the one-sided alternative is that $Y$ is stochastically greater than $X$ [7]; [8]; [9]. The Kolmogorov-Smirnov test is distribution free in the sense that if $H_{0}$ is true, the significance level does not depend on $F$ and $G$ [5].

The generalization of the classical Kolmogorov-Smirnov test is appropriate to analyse random samples defined in two or three dimensions [10]. The Kolmogorov-Smirnov test for two independent samples was developed by Smirnov (1939). When a non-directional and two tailed alternative hypothesis is evaluated, Kolmogorov-Smirnov test for two independent samples is sensitive to any kind of distributional difference (i.e., a difference with respect to location/central tendency, dispersion/variability, skewness, and kurtosis). When a directional and one-tailed alternative hypothesis is evaluated, the test evaluates the relative magnitude of the scores in the two distributions [11]; [12]; [13]; [14]. The Kolmogorov-Smirnov test has at least two major advantages over the chi- square test:

- It can be used with small sample sizes, where the validity of the chi- square test would be questionable.

- Often it appears to be a more powerful test than the chi-square test for any sample size [15]; [16]; [17].

The Kolmogorov-Smirnov test statistic $D_{n}$, is defined by $D_{n}=\sup \left|F(x)-F_{n}(x)\right|$, where $n$ is the sample size, $F(x)$ is a hypothesized cumulative distribution function with fixed parameters, and $F_{n}(x)$ is a step-function that increases by $1 / n$ at each data value [18]. By the Glivenko-Cantelli Theorem, $D_{n}$ converges to 0 almost surely under $H_{0}$ [19]. Computations of the test statistics for the Kolmogorov-Smirnov test for two independent samples involve the comparison of two cumulative frequency distributions. Whereas the Kolmogorov-Smirnov goodness-of-fit test for a single sample compares the cumulative frequency distribution of a single sample with a hypothesized theoretical or empirical cumulative frequency distribution, the Kolmogorov-Smirnov test for two independent samples compares the cumulative frequency distributions of two independent samples. If, in fact, the two samples 
are derived from the same population, the two cumulative frequency distributions would be expected to be identical or reasonably similar to one another [14]. The order statistics corresponding to two random samples of size $m$ and $n$ from continuous populations $F_{X}$ and $F_{Y}$, are

$$
X_{(1)}, X_{(2)}, \ldots, X_{(m)} \text { and } Y_{(1)}, Y_{(2)}, \ldots, Y_{(n)}
$$

Their respective empirical distribution functions, denoted by $S_{m}(x)$ and $S_{n}(x)$, are defined as:

$$
\begin{aligned}
& S_{m}(x)= \begin{cases}0 & \text { if } \quad \mathrm{x}<X_{(1)} \\
k / m & \text { if } X_{(k)} \leq x<X_{(k+1)} \text { for } k=1,2, \ldots, m-1 \\
1 & \text { if } \mathrm{x} \geq X_{(m)}\end{cases} \\
& S_{n}(x)= \begin{cases}0 & \text { if } \mathrm{x}<Y_{(1)} \\
k / n & \text { if } Y_{(k)} \leq x<Y_{(k+1)} \text { for } k=1,2, \ldots, n-1 \\
1 & \text { if } \quad \mathrm{x} \geq Y_{(n)}\end{cases}
\end{aligned}
$$

In a combined ordered arrangement of the $m+n$ sample observations, $S_{m}(x)$ and $S_{n}(x)$ are the respective proportions of $X$ and $Y$ observations which do not exceed the specific value of $x$. If the null hypothesis $H_{0}: F_{Y}(x)=F_{X}(x)$ for all $x$ is true, the population distributions are identical and we have two samples from the sample populations. The empirical distribution functions for the $X$ and $Y$ samples are reasonable estimates their respective population cumulative distribution function. Therefore, allowing for sampling variation, there should be reasonable agreement between the two empirical distributions if needed $H_{0}$ is true; otherwise the data suggest that $H_{0}$ is not true and therefore should be rejected [20].

\section{Some Preliminaries}

The power of a statistical test is the probability of rejecting the null hypothesis when it is false and it depends on three factors such as the $\alpha$ level of the experimenter, sample size and the effect size [21]. The significance criterion 
represents the standard of proof that the phenomenon exists, or the risk of mistakenly reject the null hypothesis. A critical effect size is briefly is a measure of how strong the theory is minimally important for the society and it is population-specific as well as measurement-specific [22]. The relationship between the effect size and power of a statistical test can be explained as follows. The larger the effect size, when other factors are equal, the greater the power of a test [23]. Moreover, because the sensible estimation of population parameters increases with sample size, this greater sensibility will be reflected in greater statistical power to detect effects, where association is non-linear and a law of diminishing returns revisits [24].

A number of algorithms are developed for calculating the exact powers and level of significance of statistical tests when the true cumulative distribution function $F(x)$ is continuous [25]. In a priori power analysis, sample size $N$ is computed as a function of the required power level (1- $\beta)$, so the significance level and the population effect size to be detected with this power level. In contrast, post hoc power analyses compute $(1-\beta)$ as a function of significance level, the population effect size parameter, and sample size(s) of the study. Compromise power analyses, compute both significance and the power level as functions of the effect size, and the error probability ratio, $q=\beta / \alpha$; while sensitivity analyses compute critical population effect size as a function of $\alpha, \beta$ and sample size. Finally criterion analyses compute $\alpha$, as a function of $1-\beta$, the effect size and the given sample size [26].

Type I error is frequently assigned by many researchers as the fourth factor effecting statistical power of a test. A Type I error occurs when a true null hypothesis is rejected and the likelihood of committing a Type I error is specified by alpha level being employed in evaluation process. The researcher should employ the lower values of alpha level to eliminate this error [14]. Meanly, Type I error refers to the probability of rejecting the null hypothesis when it is true [21]. The choice of a particular risk level for making a Type I error is dependent on the cost of making a Type I error [27]. Monte Carlo procedures enable to estimate the Type I comparisonwise and experimentwise error rates for multiple comparison procedures used for pairwise comparisons between means [28].

Measures of skewness and kurtosis are often used to describe shape characteristic of a distribution, in tests of normality and in studies of robustness to normal theory [29]. A skewed distribution is neither symmetric nor normal because the data values trail off more sharply on one side than on the other. One of the fundamental problems with skewness in data is many of the most common statistical methods require at least an approximately normal distribution and when these methods are employed on skewed data, the outcomes may well be misleading or just plain wrong. Even when the answers are basically correct, there is often some efficiency lost and the analysis has not made the best use of all of the information in the data set. One solution to this problem is to use transformation to make a skewed distribution more symmetric [30].

The skewness of a random variable $X$ is often measured by third central 
moment $\left(\gamma_{1}\right)$ or $\beta_{1}$ as the formulas below,

$$
\gamma_{1}=\frac{\mu_{3}}{\sigma_{3}} \quad \text { or } \quad \sqrt{\beta_{1}}=\frac{\mu_{3}}{\mu_{2}^{3 / 2}}
$$

where $\mu$ and $\sigma$ are the mean and standard deviation respectively. For the Pareto distribution with density $f(x)=\theta / x^{\theta+1}$, for $x \geq 1$ and $\theta>0$, the non-central moments are found to be $\mu_{k}^{\prime}=\theta /(\theta-k)$; therefore, this skewness coefficients becomes arbitrarily large as $\varepsilon \rightarrow 0^{+}$for $\theta=3+\varepsilon \quad$ [31]; [32].

Darlington (1970) [33] argued that kurtosis is best described as a measure of unimodality versus bimodality rather than as a measure of peakedness versus flatness, where the lower kurtosis means the greater bimodality. The fourth standardized moment of a normal distributed population, or its random variable $X$, is given by;

$$
\beta_{2}=\frac{E(X-\mu)^{4}}{\left[E(X-\mu)^{2}\right]^{2}}=\frac{E(X-\mu)^{4}}{\sigma^{4}}
$$

where $E$ is denoted by the expected value operator. This fourth moment $\left(\beta_{2}\right)$ traditionally measures kurtosis and equals to 3 for the normal distribution. Unimodal distributions which have higher peaks in the center of the distribution $\left(\beta_{2}>3\right)$ and are often described as leptokurtic [34]. Conventional but conservative alpha levels are used to evaluate significance of skewness with small to moderate samples, while for large samples, the shape of the distribution gives the researcher an opinion on skewness and kurtosis [35].

\section{Monte Carlo Simulation Study}

Monte Carlo simulation establishes to make empirical assessment of a statistic in random samples being obtained from a pseudo-population that consists of resemble samples [36]. At this point, the Monte Carlo principle may be introduced as a pioonering technique in terms of finding approximate solutions to mathematical or physical problems and by using computer-based simulation programs and random sample procedures. The idea behind the Monte Carlo methodology is the law of large numbers and the initial Monte Carlo principle performs,

- to make statistical estimation based on weak mathematical theory,

- $\quad$ to test the null hypothesis when a wide variety of possible cases are available,

- to evaluate the robustness of parametric outcomes when contradictory assumptions are available,

- to appreciate the quality of estimation methods

- $\quad$ to compare the characteristics of two or more estimators [36]; [37]. 
Additionally, Monte Carlo simulation is also employed in such circumstances that the concerning assumptions are violated or theoretical sample distributions are not presented [38].

This paper utilizes from Monte Carlo simulation and for this purpose runs SAS 9.00 computer package program. RANNOR procedure in SAS, generates random numbers from a standard normal distribution involved in Fleishman's power function with a population mean of zero, and a standard deviation of one. Fleishman's power function uses the following formula,

$$
\mathrm{Y}=\mathrm{a}+[(\mathrm{dX}+\mathrm{c}) \mathrm{X}+\mathrm{b}] \mathrm{X}
$$

where, $\mathrm{X}$ denotes a random variable with a mean of zero and a standard deviation of one, and it is generated by RANNOR, as mentioned above. Besides, Y denotes a distribution depending on constant terms; a, b, c, and d are coefficients identified with respect to different values of standard deviation, skewness and kurtosis, where coefficient a is constant. Coefficients b, c and d are introduced by Fleishman, while coefficient a and c are always opposite signed.

This paper concentrates on the distributions with highest coefficient of kurtosis (that is 3,75) in Fleishman's power function to interpret the effect of skewness on statistical power of Kolmogorov-Smirnov two-sample test when the kurtosis value is 'constant'. Fleishman's power function comprises 9 distributions with constant kurtosis value of 3,75, but different skewness values of 1,75, 1,50, $1,25,1,00,0,75,0,50,0,25,0,00$ and $-0,25$. The paper deals with totally 32 representative sample size simulation combinations, involving four small and equal; twelve small and different; four large and equal; and twelve large and different sample sizes. Table 1 summarizes these combinations. The paper regards the standard deviation ratios as $\sigma_{1}: \sigma_{2}=2, \sigma_{1}: \sigma_{2}=3$ and $\sigma_{1}: \sigma_{2}=4$ in $\alpha=0,05$ significance level, in this way $1152(9 \times 32 \times 4)$ syntaxes are written and 30.000 repetitions are performed for every syntaxes.

\section{Simulation Results}

The simulation results show similarities among 9 distributions of the study, such that in all distributions and combinations, when kurtosis value is constant, decreasing the coefficients of skewness does not affect the probability of Type I error rates for KS-2 test, where all these rates are less than $\alpha=0,05$ significance level. Again, for all distributions, the results indicate that statistical power of KS-2 test increases, when sample sizes increase; therefore standard deviation ratios have favorably effects as well. The most remarkable outcome of the simulation study is the decrease of the statistical power for KS-2 test, with respect to a decrease on the coefficient of skewness $\left(\gamma_{1}\right)$. In that case, for all sample sizes and standard deviation ratios, the most significant loss of power is recognized when the coefficient of skewness is decreased from $\gamma_{1}=1,75$ to $\gamma_{1}=1,50$. In addition, statistical power of KS-2 test increases when both the coefficient of skewness and 
standard deviation ratios increase. The most significant increase on statistical power of KS-2 test is recognized when standard deviation ratio increases from 2 to 3. For both large and small sample sizes; Table 3, Table 4, Table 5 and Table 6 represent the observed values of statistical power of KS-2 test with respect to a decrease on the coefficient of skewness.

Table 1: Sample Size Combinations Being Used in Monte Carlo Simulation

\begin{tabular}{|l|l|}
\hline Sample Size & Sample Size Combinations \\
\hline Small and Equal & $(5,5),(10,10),(15,15),(20,20)$ \\
\hline Small and Different & $\begin{array}{l}(5,10),(5,15),(5,20),(10,5),(10,15),(10,20), \\
(15,5),(15,10),(15,20),(20,5),(20,10),(20,15)\end{array}$ \\
\hline Large and Equal & $(25,25),(50,50),(75,75),(100,100)$ \\
\hline Large and Different & $(100,25),(100,50),(100,75)$ \\
\hline
\end{tabular}

Table 2: Fleishman’s Power Function

\begin{tabular}{lccccc}
\hline $\begin{array}{c}\text { Skewness } \\
\left(\gamma_{1}\right)\end{array}$ & $\begin{array}{c}\text { Kurtosis } \\
\left(\gamma_{2}\right)\end{array}$ & a & b & c & d \\
\hline 1.75 & 3.75 & -0.3994966745 & 0.9296605248 & 0.3994966745 & -0.0364669928 \\
\hline 1.50 & 3.75 & -0.2210276210 & 0.8658862035 & 0.2210276210 & 0.0272206992 \\
\hline 1.25 & 3.75 & $-0,1606425556$ & 0,8188815613 & 0,1606425556 & 0,0491651717 \\
\hline 1.00 & 3.75 & $-0,1194238366$ & 0,7894207442 & 0,1194238366 & 0,0615396192 \\
\hline 0.75 & 3.75 & $-0,0856305956$ & 0,7699520206 & 0,0856305956 & 0,0693485545 \\
\hline 0.50 & 3.75 & $-0,0555244412$ & 0,7573998478 & 0,0555244412 & 0,0742591514 \\
\hline 0.25 & 3.75 & $-0,0273411959$ & 0,7503153411 & 0,0273411959 & 0,0769928241 \\
\hline 0.00 & 3.75 & 0.00 & 0,7480208079 & 0.00 & 0,0778727161 \\
\hline-0.25 & 3.75 & 0,0273411959 & 0,7503153411 & $-0,0273411959$ & 0,0769928241 \\
\hline
\end{tabular}

Source: C.H. Lee; A Monte Carlo Study of Two Nonparametric Statistics With Comparisons of Type I Error Rates and Power, Unpublished PhD. Dissertation, Oklahoma State University, 2007, p.173-174. [39] 
Table 3: The Probabilities of Type I Error Rates of KS-2 Test for Small and Large Sample Sizes

\begin{tabular}{|c|c|c|c|c|c|c|c|c|c|c|c|}
\hline \multirow{2}{*}{$\gamma_{2}$} & \multirow{2}{*}{$\mathrm{n}_{1}$} & \multirow{2}{*}{$\mathrm{n}_{2}$} & \multicolumn{9}{|c|}{ The Probabilities of Type I Error Rates of KS-2 Test for Small Sample Sizes. } \\
\hline & & & $\gamma_{1=1,75}$ & $\gamma_{1=1,50}$ & $\gamma_{1=1,25}$ & $\gamma_{1=1,00}$ & $\gamma_{1=0,75}$ & $\gamma_{1=0,50}$ & $\gamma_{1=0,25}$ & $\gamma_{1=0,00}$ & $\gamma_{1=-0,25}$ \\
\hline \multirow{16}{*}{3,75} & 5 & 5 & 0,007 & 0,007 & 0,009 & 0,009 & 0,009 & 0,008 & & 0,008 & 0,008 \\
\hline & 5 & 10 & 0,020 & 0,018 & 0,020 & 0,020 & 0,019 & 0,020 & 0,018 & 017 & 0,020 \\
\hline & 5 & 15 & 0,023 & 0,023 & 0,024 & 0,023 & 0,022 & 0,024 & 0,025 & 023 & 0,024 \\
\hline & 5 & 20 & 0,023 & 0,023 & 0,026 & 0,026 & 0,024 & 0,026 & 0,024 &, 027 & 0,027 \\
\hline & 10 & 5 & 0,020 & 0,020 & 0,020 & 0,020 & & & & 020 & 0,018 \\
\hline & 10 & 10 & 0,012 & 0,01 & & 0,012 & & & & & 0,012 \\
\hline & 10 & 15 & 0,030 & 0,0 & & 0,029 & & & & & 0,029 \\
\hline & 10 & 20 & 0,030 & 0,030 & & 0,030 & & & & & 0,030 \\
\hline & 15 & 5 & 0,024 & & & 0,023 & & & & & 0,025 \\
\hline & 15 & 10 & & & & 0,030 & & & & & 0,029 \\
\hline & 15 & 15 & & & & 6 & & & & & 0,027 \\
\hline & 15 & 20 & 0,034 & & & 0,034 & & & & & 0,033 \\
\hline & 20 & 5 & 0,025 & 0 & & 0,026 &, 026 & 26 & 4 & & 0,025 \\
\hline & 20 & 10 & 0,031 & 0,030 & 0,028 & 0,027 & 0,029 & 0,0 & 0,02 & 27 & 0,032 \\
\hline & 20 & 15 & 0,034 & 0,03 & & 0,034 & & 0,0 & 6 & 34 & 0,035 \\
\hline & 20 & 20 & & 0,034 & 0,034 & 0,032 & 0,031 & 0,033 & 0,037 & 0,033 & 0,034 \\
\hline \multirow{2}{*}{$\gamma_{2}$} & \multirow{2}{*}{$\mathrm{n}_{1}$} & \multirow[b]{2}{*}{$\mathrm{n}_{2}$} & \multicolumn{9}{|c|}{ The Probabilities of Type I Error Rates of KS-2 Test for Large Sample Sizes } \\
\hline & & & $\gamma_{1=1,75}$ & $\gamma_{1=1,50}$ & $\gamma_{1=1,25}$ & $\gamma_{1=1,00}$ & $\gamma_{1=0,75}$ & $\gamma_{1=0,50}$ & $\gamma_{1=0,25}$ & $\gamma_{1=0,00}$ & $\gamma_{1=-0,2}$ \\
\hline \multirow{16}{*}{3,75} & 25 & 25 & 0,033 & 0,034 & 0,035 & 0,039 & 0,038 & 0,037 & 0,034 & 0,037 & 0,035 \\
\hline & 25 & 50 & 0,038 & 0, & & 0,038 & & & & & 0,039 \\
\hline & 25 & 75 & 0,038 & 0,03 & 0,036 & 0,038 & 0,038 & 0,037 & 0,038 & 0,037 & 0,039 \\
\hline & 25 & 100 & 0,037 & 0,03 & & 0,035 & & & & & 0,037 \\
\hline & 50 & 25 & 0,038 & 0,036 & & 0,037 & & & & & 0,037 \\
\hline & 50 & 50 & 0,037 & & & 0,037 & & & & & 0,040 \\
\hline & 50 & 75 & 0,039 & 0,037 & & 0,039 & & & & 038 & 0,037 \\
\hline & 50 & 100 & 0,041 & 0,03 & & 0,042 & 0,039 & & 0,0 & 0,040 & 0,040 \\
\hline & 75 & 25 & 0,038 & 0,037 & & 0,038 & 0,039 & 0,038 & 0,038 & 0,037 & 0,038 \\
\hline & 75 & 50 & 0,039 & 0,037 & 0,037 & 0,038 & 0,038 & 0,037 & 0,038 & 0,036 & 0,036 \\
\hline & 75 & 75 & 0,044 & 0,0 & & 0,044 & 0,043 & 0,0 & 0,040 & 0,042 & 0,042 \\
\hline & 75 & 100 & 0,039 & 0,03 & 0,039 & 0,039 & 0,0 & 0,0 & 0,038 & 0,041 & 0,039 \\
\hline & 100 & 25 & 0,037 & 0,0 & & 0,036 & & 0,0 & 0,039 & 0,037 & 0,037 \\
\hline & 100 & 50 & 0,040 & 0,0 & & 0,038 & & 0,0 & & 0,0 & 0,041 \\
\hline & 100 & 75 & 0,042 & 0,041 & 0,041 & 0,041 & 0,039 & 0,040 & 0,038 & 0,040 & 0,039 \\
\hline & 100 & 100 & 0,037 & 0,035 & 0,036 & 0,038 & 0,037 & 0,036 & 0,034 & 0,035 & 0,034 \\
\hline
\end{tabular}


Table 4: The Statistial Power of KS-2 Test for Small and Large Sample Sizes when $\sigma_{1}: \sigma_{2}=2$

\begin{tabular}{|c|c|c|c|c|c|c|c|c|c|c|c|}
\hline \multirow[b]{2}{*}{$\gamma_{2}$} & \multirow{2}{*}{$\mathrm{n}_{1}$} & \multirow{2}{*}{$\mathrm{n}_{2}$} & \multicolumn{9}{|c|}{ The Statistical Rower Values of KS-2 Test for Small Sample Sizer } \\
\hline & & & $\gamma_{1=1,75}$ & $\gamma_{1=1,50}$ & $\gamma_{1=1,25}$ & $\gamma_{1=1,00}$ & $\gamma_{1=0,75}$ & $\gamma_{1=0,50}$ & $\gamma_{1=0,25}$ & $\gamma_{1=0,00}$ & $\gamma_{1=-0,25}$ \\
\hline \multirow{16}{*}{3,75} & 5 & 5 & 0,029 & 0,013 & 0,014 & 0,012 & 0,012 & 0,012 & 0,010 & 0,010 & 0,010 \\
\hline & 5 & 10 & 0,157 & 0,076 & 0,059 & 0,051 & & 0,047 & 0,045 & & 46 \\
\hline & 5 & 15 & 0,162 & 0,094 & 0,077 & 0,069 & 0,067 & 0,062 & 0,061 & 0,063 & 0,059 \\
\hline & 5 & 20 & 73 & 0,102 & 0,087 & 0,075 & 0,074 & 0,073 & 0,071 & 0,070 & 0,072 \\
\hline & 10 & 5 & 0,049 & 0,026 & 0,019 & 0,018 & 0,017 & 0,017 & 0,018 & 0,016 & 0,017 \\
\hline & 10 & 10 & 24 & 0,043 & 0,032 & 0,030 & 0,026 & 0,024 & 0,024 & 0,024 & 0,023 \\
\hline & 10 & 15 & & 0,119 & 0,091 & 0,079 & 0,075 & 0,067 & 0,068 & 0,067 & 0,066 \\
\hline & 10 & 20 & 0,306 & 0,139 & 0,112 & 0,093 & 0,091 & 0,084 & 0,081 & 0,080 & 0,081 \\
\hline & 15 & 5 & 0,051 & 0,024 & 0,020 & 0,019 & 0,018 & 0,017 & 0,016 & 0,018 & 0,018 \\
\hline & 15 & 10 & 0,221 & 0,082 & 0,065 & 0,056 & 0,052 & 0,048 & 0,046 & 0,046 & 0,046 \\
\hline & 15 & 15 & & & & 0,080 & 071 & 062 & 0,065 & & 0,063 \\
\hline & 15 & 20 & 0,579 & 0,225 & 0,151 & 0,126 & 0,114 & 0,107 & 0,105 & & 0,101 \\
\hline & 20 & 5 & 0,047 & 0,023 & 0,020 & 0,017 & 0,017 & 0,016 & 0,015 & 017 & 0,016 \\
\hline & 20 & 10 & 0,294 & 0,089 & 0,063 & 0,056 & 0,049 & 0,046 & 0,046 & 0,043 & 0,044 \\
\hline & 20 & 15 & 0,449 & 0,155 & 0,111 & 0,091 & 0,081 & 0,077 & 0,073 & 0,075 & 0,074 \\
\hline & 20 & 20 & 25 & 0,231 & 0,155 & 0,126 & 0,111 & 0,105 & 0,097 & 0,095 & 0,101 \\
\hline & & & \multicolumn{9}{|c|}{ The Statistical Power Values of KS-2 Test for Large Sample Sizes } \\
\hline$i_{2}$ & ${ }_{1}$ & $\mathrm{I}_{2}$ & $\gamma_{1=1,75}$ & $\gamma_{1=1,50}$ & $\gamma_{1=1,25}$ & $\gamma_{1=1,00}$ & $\gamma_{1=0,75}$ & $\gamma_{1=0,50}$ & $\gamma_{1=0,25}$ & $\gamma_{1=0,00}$ & $\gamma_{1=-0,}$ \\
\hline \multirow{16}{*}{3,75} & 25 & 25 & 0,785 & 0,318 & 0,206 & 0,169 & 0,146 & 0,134 & 0,122 & 0,130 & 0,125 \\
\hline & 25 & 50 & 0,894 & 0,477 & 0,329 & 0,271 & 0,236 & 0,218 & 0,206 & 0,198 & 0,204 \\
\hline & 25 & 75 & 52 & 0,549 & 0,3 & 0,314 & 0,277 & 0,253 & 0,238 & 0,239 & 0,243 \\
\hline & 25 & 100 & 0,954 & 0,584 & 0,404 & 0,336 & 0,296 & 0,275 & 0,267 & 0,254 & 0,260 \\
\hline & 50 & 25 & 0,967 & 0,456 & 0,274 & 0,217 & 0,182 & 0,160 & 0,150 & 0,147 & 0,157 \\
\hline & 50 & 50 & 0,997 & 0,735 & 0,501 & 0,398 & 0,348 & 0,310 & 0,291 & 0,289 & 0,292 \\
\hline & 50 & 75 & 0,999 & 0,821 & 0,600 & 0,494 & 0,434 & 0,392 & 0,373 & 0,366 & 0,370 \\
\hline & 50 & 100 & 1,000 & 0,877 & 0,6 & 0,579 & 0,509 & 0,465 & 0,445 & 138 & 0,443 \\
\hline & 75 & 25 & 0,994 & 0,541 & 0,310 & 0,232 & 0,193 & 0,173 & 0,159 & 0,157 & 0,161 \\
\hline & 75 & 50 & 00 & 0,849 & 0,597 & 0,463 & 0,403 & 0,363 & 0,340 & 0,331 & 0,336 \\
\hline & 75 & 75 & & 0,946 & 0,7 & 0,648 & 0,56 & 0,526 & 0,497 & 0,485 & 0,493 \\
\hline & 75 & 100 & 1,000 & 0,967 & 0,8 & 0,712 & 0,646 & 0,595 & 0,566 & 0,552 & 0,562 \\
\hline & 100 & 25 & 0,999 & 0,582 & 0,3 & 0,242 & 0,200 & 0,175 & 0,159 & 0,157 & 0,163 \\
\hline & 100 & 50 & 1,000 & 0,920 & 0,686 & 0,551 & 0,471 & 0,421 & 0,391 & 0,388 & 0,389 \\
\hline & 100 & 75 & 1,000 & 0,976 & 0,831 & 0,713 & 0,635 & 0,584 & 0,554 & 0,541 & 0,553 \\
\hline & 100 & 100 & 1,000 & 0,990 & 0,887 & 0,784 & 0,714 & 0,662 & 0,633 & 0,626 & 0,633 \\
\hline
\end{tabular}


Table 5: The Statistial Power of KS-2 Test for Small and Large Sample Sizes When $\sigma_{1}: \sigma_{2}=3$

\begin{tabular}{|c|c|c|c|c|c|c|c|c|c|c|c|}
\hline \multirow{2}{*}{$\gamma_{2}$} & \multirow{2}{*}{$n_{1}$} & \multirow{2}{*}{$\mathrm{n}_{2}$} & \multicolumn{9}{|c|}{ The Statistical Power Values of KS-2 Test for Small Sample Sizes. } \\
\hline & & & $\gamma_{1=1,75}$ & $\gamma_{1=1,50}$ & $\gamma_{1=1,25}$ & $\gamma_{1=1,00}$ & $\gamma_{1=0,75}$ & $\gamma_{1=0,50}$ & $\gamma_{1=0,25}$ & $\gamma_{1=0,00}$ & $\gamma_{1=-0,25}$ \\
\hline \multirow{16}{*}{3,75} & 5 & 5 & 0,046 & 0,026 & 0,022 & 0,018 & 0,017 & 0,017 & 0,015 & 0,015 & 0,014 \\
\hline & 5 & 10 & 0,234 & 0,137 & 0,107 & 0,094 & 0,086 & 0,080 & 0,079 & 0,080 & 0,079 \\
\hline & 5 & 15 & 0,243 & 0,162 & 0,136 & 0,119 & 0,113 & 0,106 & 0,101 & 0,103 & 0,105 \\
\hline & 5 & 20 & 0,254 & 0,176 & 0,150 & 0,133 & 0,126 & 0,117 & 0,116 & 0,114 & 0,120 \\
\hline & 10 & 5 & 0,090 & 0,044 & 0,032 & 0,029 & 0,024 & 0,021 & 0,024 & 0,023 & 0,024 \\
\hline & 10 & 10 & 0,228 & 0,105 & 0,074 & 0,057 & 0,053 & 0,046 & 0,042 & 0,043 & 0,042 \\
\hline & 10 & 15 & 0,458 & 0,257 & 0,185 & 0,157 & 0,142 & 0,133 & 0,122 & 0,127 & 0,125 \\
\hline & 10 & 20 & 0,470 & 0,283 & 0,223 & 0,190 & 0,173 & 0,161 & 0,152 & 0,155 & 0,156 \\
\hline & 15 & 5 & 0,103 & 0,044 & 0,032 & 0,027 & 0,025 & 0,024 & 0,023 & 0,019 & 0,019 \\
\hline & 15 & 10 & 0,397 & 0,192 & 0,132 & 0,111 & 0,092 & 0,085 & 0,084 & 0,085 & 0,084 \\
\hline & 15 & 15 & 0,593 & 0,313 & 0,221 & 0,179 & 0,158 & 0,139 & 0,131 & 0,130 & 0,131 \\
\hline & 15 & 20 & 0,782 & 0,491 & 0,359 & 0,297 & 0,256 & 0,235 & 0,229 & 0,221 & 0,225 \\
\hline & 20 & 5 & 0,107 & 0,043 & 0,030 & 0,022 & 0,021 & 0,020 & 0,019 & 0,016 & 0,019 \\
\hline & 20 & 10 & 0,514 & 0,236 & 0,157 & 0,128 & 0,101 & 0,095 & 0,089 & 0,084 & 0,087 \\
\hline & 20 & 15 & 0,692 & 0,371 & 0,261 & 0,217 & 0,185 & 0,171 & 0,159 & 0,163 & 0,164 \\
\hline & 20 & 20 & 0,837 & 0,521 & 0,376 & 0,308 & 0,270 & 0,242 & 0,229 & 0,224 & 0,230 \\
\hline \multirow{2}{*}{$\gamma_{2}$} & \multirow{2}{*}{$\mathrm{n}_{1}$} & \multirow{2}{*}{$\mathrm{n}_{2}$} & \multicolumn{9}{|c|}{ The Statistical Powet Values of KS-2 Test for Large Sample Sizes. } \\
\hline & & & $\gamma_{1=1,75}$ & $\gamma_{1=1,50}$ & $\gamma_{1=1,25}$ & $\gamma_{1=1,00}$ & $\gamma_{1=0,75}$ & $\gamma_{1=0,50}$ & $\gamma_{1=0,25}$ & $\gamma_{1=0,00}$ & $\gamma_{1=-0,25}$ \\
\hline \multirow{16}{*}{3,75} & 25 & 25 & 0,944 & 0,679 & 0,513 & 0,411 & 0,375 & 0,341 & 0,320 & 0,314 & 0,317 \\
\hline & 25 & 50 & 0,981 & 0,847 & 0,706 & 0,627 & 0,565 & 0,527 & 0,509 & 0,501 & 0,512 \\
\hline & 25 & 75 & 0,996 & 0,905 & 0,779 & 0,697 & 0,637 & 0,606 & 0,586 & 0,582 & 0,579 \\
\hline & 25 & 100 & 0,996 & 0,918 & 0,807 & 0,734 & 0,679 & 0,643 & 0,623 & 0,611 & 0,621 \\
\hline & 50 & 25 & 0,998 & 0,897 & 0,708 & 0,600 & 0,509 & 0,471 & 0,444 & 0,429 & 0,442 \\
\hline & 50 & 50 & 1,000 & 0,987 & 0,931 & 0,866 & 0,808 & 0,767 & 0,742 & 0,736 & 0,746 \\
\hline & 50 & 75 & 1,000 & 0,996 & 0,967 & 0,929 & 0,891 & 0,864 & 0,844 & 0,840 & 0,843 \\
\hline & 50 & 100 & 1,000 & 0,998 & 0,986 & 0,961 & 0,937 & 0,919 & 0,903 & 0,901 & 0,902 \\
\hline & 75 & 25 & 1,000 & 0,962 & 0,800 & 0,670 & 0,587 & 0,520 & 0,496 & 0,486 & 0,493 \\
\hline & 75 & 50 & 1,000 & 0,999 & 0,976 & 0,935 & 0,893 & 0,860 & 0,837 & 0,836 & 0,843 \\
\hline & 75 & 75 & 1,000 & 1,000 & 0,997 & 0,987 & 0,975 & 0,962 & 0,951 & 0,948 & 0,954 \\
\hline & 75 & 100 & 1,000 & 1,000 & 0,999 & 0,993 & 0,986 & 0,981 & 0,977 & 0,975 & 0,976 \\
\hline & 100 & 25 & 1,000 & 0,981 & 0,842 & 0,713 & 0,623 & 0,559 & 0,525 & 0,511 & 0,522 \\
\hline & 100 & 50 & 1,000 & 1,000 & 0,993 & 0,973 & 0,945 & 0,922 & 0,907 & 0,904 & 0,908 \\
\hline & 100 & 75 & 1,000 & 1,000 & 1,000 & 0,996 & 0,989 & 0,983 & 0,977 & 0,974 & 0,978 \\
\hline & 100 & 100 & 1,000 & 1,000 & 1,000 & 0,999 & 0,997 & 0,994 & 0,993 & 0,991 & 0,991 \\
\hline
\end{tabular}


Table 6: The Statistial Power of KS-2 Test for Small and Large Sample Sizes

$$
\text { When } \sigma_{1}: \sigma_{2}=4
$$

\begin{tabular}{|c|c|c|c|c|c|c|c|c|c|c|c|}
\hline \multirow{2}{*}{$\gamma_{2}$} & \multirow{2}{*}{$\mathrm{n}_{1}$} & \multirow{2}{*}{$\mathrm{n}_{2}$} & \multicolumn{9}{|c|}{ The Statistical Power of KS-2 Test for Small Sample Sizes. } \\
\hline & & & $\gamma_{1=1,75}$ & $\gamma_{1=1,50}$ & $\gamma_{1=1,25}$ & $\gamma_{1=1,00}$ & $\gamma_{1=0,75}$ & $\gamma_{1=0,50}$ & $\gamma_{1=0,25}$ & $\gamma_{1=0,00}$ & $\gamma_{1=-0,25}$ \\
\hline \multirow{16}{*}{3,75} & 5 & 5 & 0,059 & 0,037 & 0,026 & 0,026 & 0,022 & 0,022 & 0,020 & 0,018 & 0,019 \\
\hline & 5 & 10 & 0,281 & 0,194 & 0,155 & 0,130 & 0,124 & 0,116 & 0,108 & 0,109 & 0,108 \\
\hline & 5 & 15 & 0,295 & 0,210 & 0,182 & 0,165 & 0,151 & 0,143 & 0,139 & 0,139 & 0,140 \\
\hline & 5 & 20 & 0,301 & 0,225 & 0,197 & 0,178 & 0,164 & 0,158 & 0,156 & 0,153 & 0,156 \\
\hline & 10 & 5 & 0,116 & 0,058 & 0,045 & 0,038 & 0,033 & 0,028 & 0,028 & 0,028 & 0,026 \\
\hline & 10 & 10 & 0,290 & 0,155 & 0,111 & 0,089 & 0,075 & 0,069 & 0,067 & 0,064 & 0,067 \\
\hline & 10 & 15 & 0,540 & 0,349 & 0,267 & 0,233 & 0,206 & 0,191 & 0,190 & 0,179 & 0,185 \\
\hline & 10 & 20 & 0,549 & 0,386 & 0,312 & 0,268 & 0,256 & 0,236 & 0,230 & 0,225 & 0,221 \\
\hline & 15 & 5 & 0,147 & 0,068 & 0,048 & 0,035 & 0,032 & 0,028 & 0,026 & 0,024 & 0,025 \\
\hline & 15 & 10 & 0,481 & 0,278 & 0,205 & 0,168 & 0,143 & 0,131 & 0,122 & 0,121 & 0,124 \\
\hline & 15 & 15 & 0,686 & 0,447 & 0,334 & 0,271 & 0,246 & 0,221 & 0,211 & 0,206 & 0,211 \\
\hline & 15 & 20 & 0,865 & 0,661 & 0,521 & 0,452 & 0,409 & 0,372 & 0,355 & 0,354 & 0,360 \\
\hline & 20 & 5 & 0,162 & 0,063 & 0,043 & 0,033 & 0,029 & 0,025 & 0,023 & 0,024 & 0,023 \\
\hline & 20 & 10 & 0,626 & 0,355 & 0,246 & 0,197 & 0,165 & 0,147 & 0,136 & 0,136 & 0,135 \\
\hline & 20 & 15 & 0,787 & 0,534 & 0,405 & 0,332 & 0,290 & 0,268 & 0,253 & 0,250 & 0,261 \\
\hline & 20 & 20 & 0,905 & 0,695 & 0,555 & 0,476 & 0,428 & 0,391 & 0,367 & 0,364 & 0,368 \\
\hline \multirow[b]{2}{*}{$\gamma_{2}$} & \multirow[b]{2}{*}{$\mathrm{n}_{1}$} & \multirow[b]{2}{*}{$\mathrm{n}_{2}$} & \multicolumn{9}{|c|}{ The Statistical Power of KS-2 Test for Large Sample Sizes } \\
\hline & & & $\gamma_{1=1.75}$ & $\gamma_{1=1.50}$ & $\gamma_{1=1.25}$ & $\gamma_{1=100}$ & $\gamma_{1=0.75}$ & $\gamma_{1=0.50}$ & $\gamma_{1=0.25}$ & $\gamma_{1=0,00}$ & $\gamma_{1=-0,25}$ \\
\hline \multirow{16}{*}{3,75} & 25 & 25 & 0,977 & 0,851 & 0,719 & 0,632 & 0,578 & 0,540 & 0,510 & 0,503 & 0,514 \\
\hline & 25 & 50 & 0,996 & 0,953 & 0,888 & 0,834 & 0,788 & 0,764 & 0,745 & 0,736 & 0,746 \\
\hline & 25 & 75 & 0,999 & 0,980 & 0,932 & 0,890 & 0,855 & 0,826 & 0,811 & 0,811 & 0,812 \\
\hline & 25 & 100 & 0,999 & 0,984 & 0,948 & 0,913 & 0,883 & 0,858 & 0,845 & 0,841 & 0,850 \\
\hline & 50 & 25 & 1,000 & 0,982 & 0,918 & 0,843 & 0,786 & 0,742 & 0,709 & 0,701 & 0,711 \\
\hline & 50 & 50 & 1,000 & 1,000 & 0,993 & 0,980 & 0,968 & 0,954 & 0,943 & 0,940 & 0,944 \\
\hline & 50 & 75 & 1,000 & 1,000 & 0,998 & 0,995 & 0,989 & 0,983 & 0,980 & 0,977 & 0,980 \\
\hline & 50 & 100 & 1,000 & 1,000 & 1,000 & 0,998 & 0,996 & 0,995 & 0,992 & 0,991 & 0,993 \\
\hline & 75 & 25 & 1,000 & 0,997 & 0,966 & 0,912 & 0,856 & 0,810 & 0,783 & 0,776 & 0,785 \\
\hline & 75 & 50 & 1,000 & 1,000 & 0,999 & 0,996 & 0,992 & 0,986 & 0,983 & 0,983 & 0,983 \\
\hline & 75 & 75 & 1,000 & 1,000 & 1,000 & 1,000 & 0,999 & 0,998 & 0,998 & 0,998 & 0,998 \\
\hline & 75 & 100 & 1,000 & 1,000 & 1,000 & 1,000 & 1,000 & 1,000 & 1,000 & 1,000 & 0,999 \\
\hline & 100 & 25 & 1,000 & 1,000 & 0,980 & 0,938 & 0,891 & 0,854 & 0,821 & 0,812 & 0,824 \\
\hline & 100 & 50 & 1,000 & 1,000 & 1,000 & 0,999 & 0,998 & 0,996 & 0,994 & 0,995 & 0,995 \\
\hline & 100 & 75 & 1,000 & 1,000 & 1,000 & 1,000 & 1,000 & 1,000 & 1,000 & 0,999 & 1,000 \\
\hline & 100 & 100 & 1,000 & 1,000 & 1,000 & 1,000 & 1,000 & 1,000 & 1,000 & 1,000 & 1,000 \\
\hline
\end{tabular}

\section{Conclusion and Discussion}

As the Monte Carlo simulation study results suggest in this paper, when the kurtosis value is constant with its highest value of 3,75, the statistical power of KS-2 test is not significantly effected by a decrease of the coefficient of skewness. For small sample sizes, the probabilities of Type I error for KS-2 test vary between the values of $(0,007)$ and $(0,038)$. The study observes the smallest value 
of this probability when the coefficient of skewness decreases from $\gamma_{1}=1,75$ to $\gamma_{1}=1,50$ for $(5,5)$ sample size, while it obtains the highest value of Type I probability when the coefficient of skewness decreases from $\gamma_{2}=3,75$ to $\gamma_{1}=0,75$ for $(20,15)$ sample size. Similarly, for large sample sizes, the probabilities of Type I error for KS-2 test vary between the values of $(0,033)$ and $(0,044)$. The smallest Type I error probability is observed when the coefficient of skewness is $\gamma_{1}=1,75$ for $(25,25)$ sample size, meanwhile the highest value of the probability is recognized when the coefficients of skewness are $\gamma_{1}=1,75$, $\gamma_{1}=1,25$ and $\gamma_{1}=1,00$ for $(75,75)$ sample size. The probabilities of Type I error for KS-2 test are found less than $\alpha=0,05$ significance level for both small and large sample sizes.

The results of the simulation study in all distributions state that when the sample size increases, the statistical power of KS-2 test also increases. For small sample pairs, when the first sample size is smaller than the second sample size, the observed statistical power of KS-2 test is more than the corresponding power when the second sample size is smaller than the first one. For instance, the observed statistical power of KS-2 test for the sample size of $(5,10)$ is more than $(10,5)$ sample size in all distributions and standard deviations. For large sample pairs, just the opposite circumstance is observed, i.e. the observed statistical power for $(50,25)$ sample size is more than $(25,50)$ sample size. Moreover, in all distributions and sample sizes, the standard deviation has favorable effect on the statistical power and the most significant statistical power increase is observed when the standard deviation ratio increases from $\sigma_{1}: \sigma_{2}=2$ to $\sigma_{1}: \sigma_{2}=3$. The results also demonstrate that when the coefficient of skewness decreases, the statistical power of KS-2 test also decreases, except for one observation. The statistical power increases when the coefficient of skewness decreases from $\gamma_{1}=0,00$ to $\gamma_{1}=-0,25$.

In the light of all these simulation results, one may suggest that the researchers concentrating on a non-parametric two-sample test, may observe higher statistical power if they choose their samples from the distribution with the coefficient of skewness, $\gamma_{1}=1,75$, and when the coefficient of kurtosis is constant $\left(\gamma_{2}=3,75\right)$ simultaneously. Additionally, the researchers may also observe higher values of statistical power, when $\sigma_{1}: \sigma_{2}$ ratio increases. For small sample sizes, the researchers may prefer the larger values of the first sample size and for large sample sizes; they may prefer the smaller values of the first sample size in order to observe higher statistical power values, numerically. 


\section{References}

[1] R. J. Shavelson, Statistical reasoning for the behavioral sciences, Third edition, Allynand Bacon, Inc., Boston, 1996.

[2] W. P. Vogt, Dictionary of Statistics and Methodology: A Nontechnical Guide for the Social Sciences, Third edition, Sage Publications, California, 2005.

[3] J. L. Rasmussen, Parametric vs nonparametric tests on non-normal and transformed data. Unpublished PhD Dissertation, Department of psychology of the graduate school of Tulane University, New Orleans, 1983.

[4] Z. Drezner, O. Turel and D. Zerom, A modified Kolmogorov-Smirnov test for normality, Communications in Statistics-Simulation and Computation, 39(4), (2010), 693-704.

[5] R.R. Wilcox, Some practical reasons for reconsidering the Kolmogorov-Smirnov Test, British Journal of Mathematical and Statistical Psychology, 50(1), (1997), 9-20.

[6] J. Capon, On the asymptotic efficiency of the Kolmogorov-Smirnov test, Journal of the American Statistical Association, 60(311), (1965), 843-853.

[7] N. Smirnov, On the estimation of the discrepancy between empirical curves of distribution for two independent samples, Bulletin Mathematique de l'Universite de Moscou, 2, (1939), 3-14.

[8] M. H. Gail and S. B. Green, Critical values for the one-sided two-sample Kolmogorov-Smirnov statistic, Journal of the American Statistical Association, 71(355), (1976), 757-760.

[9] R.H.C. Lopes, I. Reid and P.R. Hobson, The two-dimensional Kolmogorov-Smirnov test, Proceedings of XI International Workshop on Advanced Computing and Analysis Techniques Physics Research, April 23-27 2007, Amsterdam, Netherlands.

[10] G. Fasano and A. Franceschini, A multidimensional version of the Kolmogorov-Smirnov test, Monthly Notices of the Royal Astronomical Society, 225(1), (1987), 155-170.

[11] W. Daniel, Applied nonparametric statistics, PWS-Kent Publishing Company, Boston, 1990.

[12] L.A. Marascuilo and M. McSweeney, Nonparametric and distribution-free methods for the social sciences, Brooks/Cole Publications Co., California, 1977.

[13] S. Siegel and N.J. Castellan, Nonparametric statistics for the behavioral sciences, 2nd edition, Mc-Graw-Hill, New York, 1988.

[14] D. J. Sheskin, Hanbook of parametric and nonparametric statistical procedures, 3rd edition, Chapman \& Hall/CRC, New York, 2004.

[15] F.N. David and N.L. Johnson, The probability integral transformation when parameters are estimated from the sample, Biometrika, 35(1/2), (1948), 182-190.

[16] F.J. Massey, The Kolmogorov-Smirnov test for goodness of fit, Journal of the American Statistical Association, 46(253), (1951), 68-78. 
[17] H.W. Lilliefors, On the Kolmogorov-Smirnov test for normality with mean and variance unknown, Journal of the American Statistical Association, 62(318), (1967), 399-402.

[18] J.H. Drew, A.G. Glen and L.M. Leemis, Computing the cumulative distribution function of the Kolmogorov-Smirnov statistic, Computational Statistics \& Data Analysis, 34(1), (2000), 1-15.

[19] Z. Zhang and C. Wang, Investigating the effect of dependence on the performance of Kolmogorov-Smirnov test, Course Project, Michigan State University, December 2010.

[20] J.D. Gibbons and S. Chakraborti, Nonparametric Statistical Inference, Fourth edition, Marcel Decker Inc., New York, 2003.

[21] J.P. Stevens, Applied multivariate statistics for the social sciences, Fifth edition, Routledge, New York, 2009.

[22] H.C. Kraemer and S. Thiemann, How many subjects? Statistical power analysis in research, Sage Publications Inc., California, 1987.

[23] J. Cohen, Statistical power analysis for the behavioral sciences, Second edition, Lawrence Erlbaum Associates Inc., New Jersey, 1988.

[24] A. Davey and J. Savla, Statistical power Analysis with missing Data: A structural equation modelling approach, Routledge, New York, 2010.

[25] L.J. Gleser, Exact power of goodness-of-fit tests of Kolmogorov Type for discontinuous distributions, Journal of the American Statistical Association, 80(392), (1985), 954-958.

[26] F. Faul, E. Erdfelder, A. Lang and A. Buchner, G*Power 3: A flexible statistical power analysis program for the social, behavioral, and biomedical sciences, Behavioral Research Methods, 39(2), (2007), 175-191.

[27] M. L. Berenson, D. M. Levine and T. C. Krehbiel (2003). Basic business statistics: Concepts and applications, Ninth edition, Prentice Hall Inc., New Jersey, 2003.

[28] C. S. Bernhardson, 375: Type I error rates when multiple comparison procedures follow a significant F test of ANOVA, Biometrics, 31(1), (1975), 229-232.

[29] D.N. Joanes and C.A. Gill, Comparing measures of sample skewness and Kurtosis, Journal of the Royal Statistical Society: Series D (The Statistician), 47(1), (1998), 183-189.

[30] A.F. Siegel, Practical business statistics, Sixth edition, Academic Press, Boston, 2012.

[31] R. A. Groeneveld and G. Meeden, Measuring skewness and kurtosis, Journal of the Royal Statistical Society: Series D (The Statistician), 33(4), (1984), 391-399.

[32] J. A. Doornik and H. Hansen, An omnibus test for univariate and multivariate normality, Oxford Bulletin of Economics and Statistics, 70(1), (2008), 927-939.

[33] R. B. Darlington, Is kurtosis really "Peakedness"?, The American Statistician, 24(2), (1970), 19-22. 
[34] R. B. D’Agostino, A. Belanger and R. B. D’Agostino Jr., A suggestion for using powerful and information tests of normality, The American Statistician, 44(4), (1990), 316-321.

[35] B. G. Tabachnick and L. S. Fidell, Using multivariate statistics, Pearson Education Inc., Boston, 1996.

[36] C. Z. Mooney, Monte Carlo simulation. Sage Publications Inc., California, 1997.

[37] J. Jiang, Large sample techniques for statistics. Springer Science + Business Media, LLC., New York, 2010.

[38] X. Fan, À. Felsövályi, S. A. Sivo and S. C. Keenan, SAS for Monte Carlo studies: A guide for quantitative researchers. SAS Publishing, Ary, NC, 2003.

[39] C.H. Lee, A Monte Carlo study of two nonparametric statistics with comparisons of Type I error rates and power. Unpublished PhD Dissertation, Faculty of the Graduate College of the Oklahoma State University, Stillwater, 2007. 